\title{
Spectral Variability and Photometric Behaviour of the Symbiotic Nova PU Vul in 1985-1994
}

\author{
Yu.S. Efimov, R.E. Gershberg, I.S. Savanov, A.G. Shcherbakov, \\ V.A. Shcherbakov \\ Crimean Astrophysical Observatory, Nauchny, Crimea, Ukraine
}

We present preliminary results of observations of the $\mathrm{H} \alpha, \mathrm{He}$ I 5876 and $\mathrm{NaI}$ $\mathrm{D}$ lines profiles in spectra of PU Vul. The variability of profiles is confronted with the photometric behaviour. The observations were carried out with a CCD camera, installed in the coude spectrograph of the $2.6 \mathrm{~m}$ Shajn telescope. 68 spectra of the $\mathrm{H} \alpha$ line and 25 spectra in the region of the $\mathrm{HeI}$ and $\mathrm{NaI}$ lines were obtained.

Fig. 1 (left panel) presents the evolution of the $\mathrm{H} \alpha$ line from 1985 to 1994, displaying selected typical profiles in different years. The profiles are shown in a heliocentric wavelength scale and in logarithms of intensity. Variations of line intensity, line width, as well as radial velocity of the centre of weight of the total emission and absorption components are observed.

Stronger and more complex variability is observed in the region of the $\mathrm{HeI}$ and $\mathrm{NaI} \mathrm{D}$ lines. The strong $\mathrm{P} \mathrm{Cyg}$ type absorption in the $\mathrm{NaI} \mathrm{D}$ lines during 1985-1987 disappeared in later spectra as a result of total ionization of $\mathrm{Na}$ I (Fig. 1., right panel). Contrary to that behaviour, He I 5876 shows variations from a pure absorption profile to a very strong and wide emission profile.

Nevertheless, even more drastic fast changes of $\mathrm{H} \alpha$ emission line were observed in 1987 and 1989. Tamura et al. (1992) found an abrupt change of the $\mathrm{H} \alpha$ profile in September 1989. On September 10, the $\mathrm{H} \alpha$ line had an extremely wide wing and showed only a trace of the central absorption feature which was very strong on July 26. In 1990 August, the $\mathrm{H} \alpha$ emission revealed a similar profile to that of 1989 July. In Fig. 2 (right panel) $\mathrm{H} \alpha$ profiles compiled from Tamura et al. (1992) together with our observations are shown. It is seen that the profile obtained by us on July 21 , practically coincides with the profile on July 26 (Tamura et al. 1992, dashed line). We succeeded to reduce the time interval of these drastic changes in $\mathrm{H} \alpha$. Our subsequent observations were obtained on 1989 October 24. Already at this time, the H $\alpha$ profile had returned to its former shape (Fig. 2). According to Van Winckel et al. (1993), the former shape of $\mathrm{H} \alpha$ profile was observed only 5 days after 1989 September 10 . Thus, this dramatic event lasted not more than 5 days. Similar fast changes of the profile were observed in 1987 (Fig. 2, left panel). The shape of the profile changed from a double-peaked to a quasi-symmetrical one in less than $\mathbf{3 7}$ days and returned to the normal shape in 6 days. On 1987 August 8 only a weak trace of absorption in the blue wing of 

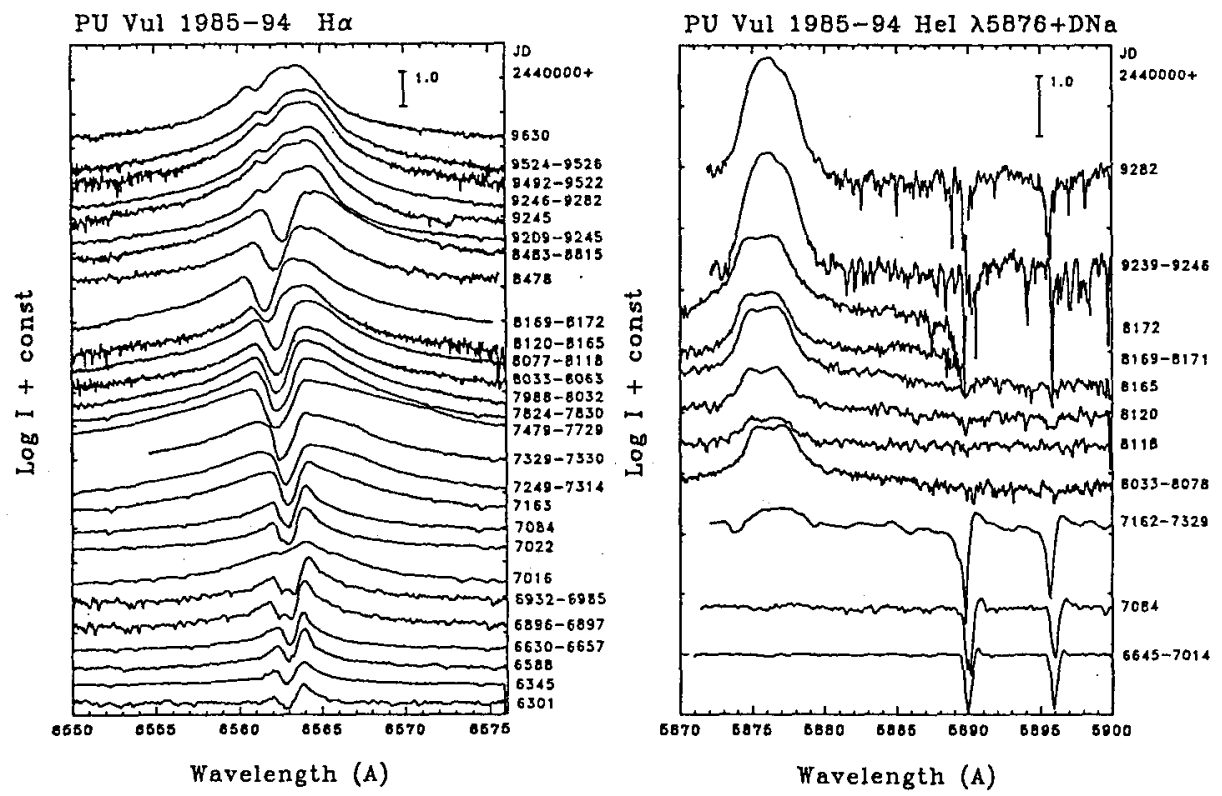

Fig. 1. Selected profiles of the H $\alpha$ line (left) and of He I 5876 and $\mathrm{NaI}$ D (right).
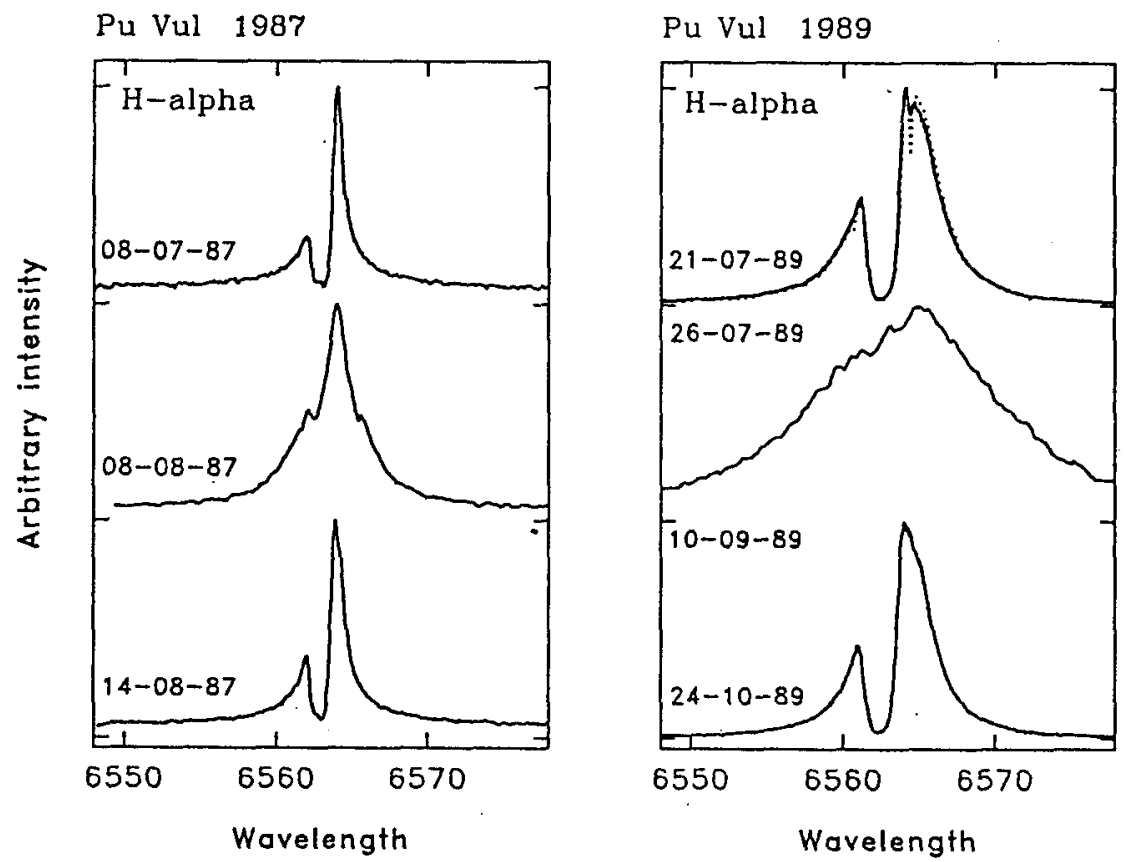

Fig. 2. Abrupt changes of the $\mathrm{H} \alpha$ profiles in 1987 and 1989 
the line is seen as compared with absorption in profiles, obtained on July 8 and on August 14. A similar weak absorption is also seen in the red wing of the line.

For a quantitative study of the character of the line variability, all observed profiles (except abnormal profiles, shown in Fig. 2) were approximated by Voigttype profiles, as shown in Fig. 3 (left panel). Three types of profiles are displayed: a moderately weak and narrow profile in 1985 , a strong and very wide profile in 1987-1992, and a very strong and moderately narrow asymmetric profile in 1993-1994. These profiles were approximated using three criteria: the shape of the blue and red wings of the line and the gradient of the growth of intensity on the red part of the profile, which is not disturbed by the absorption.

The following parameters of a line were measured: radial velocity of absorption component of the observed profile (RVabs), radial velocity of the centre of weight of emission Voigt profile (RVem), full width of the line at half maximum level (FWHM), measured on the Voigt profile, and full width of the line at the $10 \%$ of maximum level (FW0.1M).

In Fig. 3 (right panel), a comparison of measured parameters of the line and the $V$-brightness is presented. Two vertical dashed lines mark the times of drastic changes of the line profile in 1987 and 1989, as seen in Fig. 2. All measured parameters show slow changes from year to year, as well as fast variations, correlated with variations of stellar brightness.

The FWHM and the FW0.1M vary synchronously and indicate a strong growth of emission width between 1987 and 1989. The broadening of the line began at the initial stage of transition to the nebular phase, when the brightness began to drop abruptly. The width of the emission smoothly decreases while the star progressively enters the nebular phase.

The radial velocity of the total emission, corresponding to the movement of an envelope in the binary system, also shows variations from year to year with an amplitude of more than $10 \mathrm{~km} / \mathrm{s}$. The orbital elements of the system have not yet been established with certainty. According to Kanamitsu (1991), the orbital period of PU Vul must be of the order of 10 years. We have analysed our series of radial velocity measurements to search for a possible period. Several methods were applied. The highest peak in the power spectrum obtained by the Deeming method gives a period near 9.3 years. A refinement by Lafler-Kinman's and the string-length method yield a value of about $9.1 \pm 0.4$ years. The variability was also modelled by a least squares fitting of observed data by trigonometric polynomials. The period of the model was iteratively refined by the LevenbergMarquardt algorithm (Pelt 1992, Press \& Teukolsky 1988). The result of the estimate - the dashed curve in the third panel in Fig. 5 - gives an optimal period of changes of the radial velocity of about $11.2 \pm 2.2$ years.

The fast changes of the radial velocity of the total emission are surprising. These variations cannot be explained in the framework of a simple model of an expanding envelope. It was found, however, that such variability is caused by variations in the wings of the line, by their asymmetry. The model of a binary system, in which a wind outflow plays a significant role, as proposed by Kanamitsu (1991), could explain the observed variations as result of interaction 

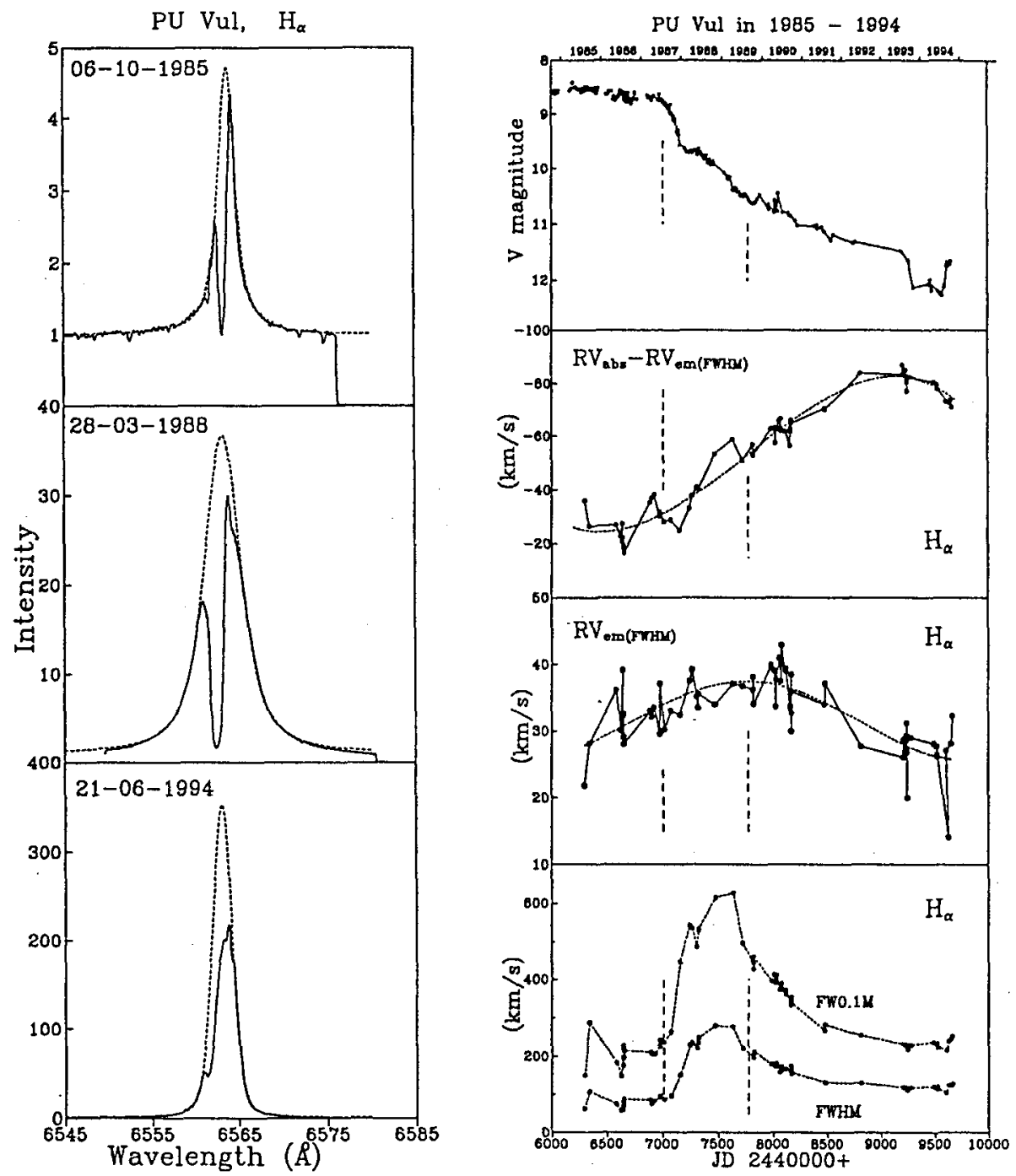

Fig. 3. Approximation of observed $\mathrm{H} \alpha$ line by Voigt profiles (left panel), and comparison between measured parameters of the $\mathrm{H} \alpha$ profiles with the $V$ band brightness of the star.

between a non-stationary wind with an expanding envelope. The correctness of such a model is confirmed by the indication of a bipolar mass outflow found by Bensammar et al. (1991), by the onset of a Wolf-Rayet wind outflow (Sion et al. 1993), and the Wolf-Rayet features observed in the spectrum of PU Vul (Tomov et al. 1991). Similar unexpected fast variations of the radial velocity were observed in the spectrum of the enigmatic binary system $\epsilon$ Aur (Gershberg 1990). 
The difference between the radial velocities of the absorption component of the line and the total emission as fitted by a Voigt profile (RVabs-RVem) determines the velocity of expansion of the envelope. Fig. 5 shows that the velocity of expansion during the time of our observations changed from $20 \mathrm{~km} / \mathrm{s}$ up to $80 \mathrm{~km} / \mathrm{s}$ (dashed curve). In 1985-1986 the velocity varied only by a few $\mathrm{km} / \mathrm{s}$, from 1986 to 1992 the envelope expanded with acceleration, and since 1992, evidence of retardation is observed. Besides the slow increase of radial velocity, rapid variations with timescales of a year and less are observed. Attention should be paid to the fact that both episodes of drastic changes of the $\mathrm{H} \alpha$ profile were followed by retardation. Tamura et al. (1992) interpret the abrupt change of the $\mathrm{H} \alpha$ profile as a result of interaction between the hot stellar wind from the hot contracting star that underwent an explosion with the cold wind from an $M$ star. However, the episodes of retardation of the envelope in 1987 and 1989 indicate that the drastic changes of the $\mathrm{H} \alpha$ profile could be as well the result of the passage of a shock wave in the expanding envelope.

Acknowledgement: This research was made possible in part by Grant R2 Q000 from the International Science Foundation.

\section{References}

Bensammar S., Friedjung M., Chauville J., Letourneur N., 1991, A\&A 245, 575

Gershberg R.E., 1990, Astron. Zh. 67, 76

Kanamitsu O., 1991, PASJ 43, 225

Pelt J., 1992, Licenced Package "Irregularly spaced data analysis", Helsinki, Finland

Press W.H., Teukolsky S.A., 1988, Computers in Physics 2, 77

Sion E.M., Shore S.N., Ready C.J., Scheible M.P., 1993, AJ 106, 2118

Tamura S., Kanamitsu O., Yamashita Y., 1992, PASJ 44, 543

Tomov T., Zamanov R., Miev L., Mikolajewski M., Georgiev L., 1991, MNRAS 252, 31

Van Winckel H., Duerbeck H.W., Schwarz H.E., 1993, A\&AS 102, 401 